\title{
Post common envelope binaries from SDSS
}

\section{II: Identification of 9 close binaries with VLT/FORS2 ${ }^{\star}$}

\author{
M. R. Schreiber ${ }^{1}$, B. T. Gänsicke ${ }^{2}$, J. Southworth ${ }^{2}$, A. D. Schwope ${ }^{3}$, and D. Koester ${ }^{4}$ \\ 1 Departamento de Física y Astronomía, Facultad de Ciencias, Universidad de Valparaíso, Valparaíso, Chile \\ e-mail: Matthias.Schreiber@uv.cl \\ 2 Department of Physics, University of Warwick, Coventry CV4 9BU, UK \\ 3 Astrophysikalisches Institut Potsdam, An der Sternwarte 16, 14482 Potsdam, Germany \\ ${ }^{4}$ Institut für Theoretische Physik und Astrophysik, University of Kiel, 24098 Kiel, Germany
}

Received 30 September 2007 / Accepted 6 March 2008

\begin{abstract}
Context. Post common envelope binaries (PCEBs) consisting of a white dwarf and a main sequence star are ideal systems to use to calibrate current theories of angular momentum loss in close compact binary stars. The potential held by PCEBs for further development of close binary evolution could so far not be exploited due to the small number of known systems and the inhomogeneity of the sample. The Sloan Digital Sky Survey is changing this scene dramatically, as it is very efficient in identifying white dwarf/main sequence (WDMS) binaries, including both wide systems whose stellar components evolve like single stars and - more interesting in the context of close binary evolution - PCEBs.

Aims. We pursue a large-scale follow-up survey to identify and characterise the PCEBs among the WDMS binaries that have been found with SDSS. We use a two-step strategy with the identification of PCEBs among WDMS binaries in the first phase and orbital period determinations in the second phase. Here we present first results of our ESO-VLT/FORS2 pilot study that targets the identification of the PCEBs among the fainter $(g \geq 18.5)$ SDSS WDMS binaries.

Methods. From published SDSS catalogues we selected 26 WDMS binaries to be observed with ESO-VLT/FORS2 in service mode. The design of the observations was to get two spectra per object separated by at least one night. We used the Na I $\lambda \lambda 8183.27,8194.81$ doublet to measure radial velocity variations of our targets and a spectral decomposition/fitting technique to determine the white dwarf effective temperatures and surface gravities, masses, and secondary star spectral types for all WDMS binaries in our sample.

Results. Among the 26 observed WDMS binaries, we find 9 strong PCEB candidates showing clear $(\geq 3 \sigma)$ radial velocity variations, and we estimate the fraction of PCEBs among SDSS WDMS binaries to be $\sim 35 \pm 12 \%$. We find indications of a dependence of the relative number of PCEBs among SDSS WDMS binaries on the spectral type of the secondary star. These results are subject to small number statistics and need to be confirmed by additional observations. Using Magellan-Clay/LDSS3, we measured the orbital periods of two PCEB candidates, SDSS J1047+0523 and SDSS J1414-0132, to be $9.17 \mathrm{~h}$ and $17.48 \mathrm{~h}$, respectively.

Conclusions. This pilot study demonstrates that our survey is highly efficient in identifying PCEBs among the SDSS WDMS binaries, and it will indeed provide the observational parameters that are needed to constrain the theoretical models of close binary evolution.
\end{abstract}

Key words. accretion, accretion disks - stars: novae, cataclysmic variables - stars: binaries: close

\section{Introduction}

The majority of all stars are born in binary or multiple star systems, and a significant fraction of these will interact at some point in their lives. Once the more massive star leaves the main sequence, and depending on the initial conditions, dynamically unstable mass transfer or a tidal instability may force the system to enter the common envelope (CE) phase (see Taam \& Ricker 2006 , for more details). In this phase a gaseous envelope around the entire binary forms, and friction within the envelope significantly reduces the binary separation. Since Paczyński (1976) it is generally believed that virtually all close compact binary systems ranging from X-ray binaries to double white dwarf binaries or cataclysmic variables, to name a few, have formed through CE evolution. Willems \& Kolb (2004) performed comprehensive binary population synthesis studies for white dwarf/main

\footnotetext{
* Based on observations collected at the European Southern Observatory, Paranal, Chile under programme ID 078.D-0719. This paper includes data gathered with the $6.5 \mathrm{~m}$ Magellan Telescopes located at Las Campanas Observatory, Chile.
}

sequence binaries (WDMS), and find that dynamically stable mass transfer may produce some additional short orbital period ( $P_{\text {orb }} \gtrsim 10$ days) systems primarily consisting of low-mass He white dwarfs $\left(0.1 \leq M_{\mathrm{wd}} \leq 0.4 M_{\odot}\right)$ and relatively massive secondary stars (up to $6 M_{\odot}$ ). In the vast majority $(\sim 75-99 \%)$ of cases, however, a CE phase is needed to produce short orbital period ( $P_{\text {orb }} \lesssim 400$ days) WDMS.

From theoretical simulations we have learned (i) that the CE phase is probably very short, i.e. $<_{\sim}^{3} \mathrm{yrs}$; (ii) that the spiral-in starts rapidly after the onset of the CE phase; and (iii) that the expected form of post-CE planetary nebular is bipolar (see e.g. Morris 1981; Taam \& Bodenheimer 1989; Taam \& Sandquist 2000; Webbink 2007, and references therein). Hydrodynamical studies have been carried out for a number of parameters (e.g. Taam \& Ricker 2006, for a recent review); however, these expensive simulations cannot be used in population synthesis models. Instead, simple equations relating the total energy of the binary before and after the CE phase are applied (e.g. Willems \& Kolb 2004). Usually, the CE phase is simply approximated by a parameterised energy (Webbink 1984; Willems \& Kolb 2004) or 
angular momentum equation (Nelemans et al. 2000). Both descriptions differ significantly in the predicted outcome of the CE phase, i.e. the energy equation predicts a much stronger shrinkage than the angular momentum equation (Nelemans \& Tout 2005). In addition, in both prescriptions the efficiency of "using" the orbital energy (or angular momentum) to expel the envelope is uncertain; hence, the $\mathrm{CE}$ phase is probably the least understood part of close binary evolution so is currently intensively debated. For recent discussions of the CE phase see Nelemans \& Tout (2005); Webbink (2007); Beer et al. (2007).

Once the envelope is expelled, the evolution of the PCEB is mainly driven by angular momentum loss (AML) due to magnetic braking. Unfortunately, the two currently favoured prescriptions for magnetic braking (Verbunt \& Zwaan 1981; Andronov et al. 2003) differ by up to two orders of magnitude in the predicted angular momentum loss rate. Even worse, it is not clear whether magnetic braking is present in all PCEBs or whether it terminates in systems where the secondary star is fully convective. To explain the orbital period gap observed in the period distribution of CVs, one in fact needs to assume the latter, known as "disrupted magnetic braking", (e.g. King 1988; Howell et al. 2001). In contrast to this, observations of single low-mass stars do not show any evidence of such a discontinuity (e.g. Pinsonneault et al. 2002).

Significant progress in our understanding of the CE phase and AML in PCEBs certainly requires both continuous theoretical efforts and innovative observational input. The potential that a large sample of white dwarf plus main sequence PCEBs could provide in this context was first realised by Schreiber \& Gänsicke (2003). They show that it is possible to determine the orbital period the PCEB had when it left the CE phase and the white dwarf temperature, as well as the orbital period, it will have when entering the next phase of interaction (becoming a cataclysmic variable). More recently, Politano \& Weiler (2006) have shown that the disrupted magnetic braking hypothesis can be tested by knowing the relative number of PCEBs among white dwarf/main sequence (WDMS) binaries as a function of secondary spectral type. Finally, Schreiber et al. (2007) recently concluded that a representative sample of PCEBs could probably constrain the $\mathrm{CE}$ efficiency, the description of the $\mathrm{CE}$ phase, and the strength of magnetic braking.

Unfortunately, only a very limited number of PCEBs with known effective temperature and orbital period is currently available. The PCEB sample that Schreiber \& Gänsicke (2003) analysed consisted of only the 30 well-studied short-period WDMS known in 2002. Since then Gänsicke et al. (2004), MoralesRueda et al. (2005), van den Besselaar et al. (2007), Shimansky et al. (2006), Aungwerojwit et al. (2007), and Tappert et al. (2007), among others, added additional systems and the current number of well studied-PCEBs is close to 50. The number of systems required to constrain close binary evolution is certainly much higher. Schreiber et al. (2007) estimate that about 200 PCEBs are needed with known white dwarf temperatures, secondary star parameters, and orbital periods.

The Sloan Digital Sky Survey (SDSS) is currently opening a new era of observational population studies for a wide variety of astronomical objects, including WDMS binaries. As by-product of the quasar search within SDSS, more than 1000 WDMS binaries have already been identified within Data Release 5 (Eisenstein et al. 2006; Silvestri et al. 2007; Southworth et al. 2007). Though very large, this set of WDMS binaries is still subject to selection effects, as it favours systems with bluish colours, i.e. hot white dwarfs. To compensate for this, we initiated a dedicated WDMS identification programme as part of
SDSS II-SEGUE, which targets systems with a strong contribution by the companion star (see Schreiber et al. 2007). In parallel, we started the identification of PCEBs among the WDMS binaries identified by SDSS by means of a radial velocity survey. Rebassa-Mansergas et al. (2007, henceforth Paper I) present a first set of 18 PCEB candidates from radial velocity variations detected among multiple SDSS spectra. In this paper we present VLT/FORS2 observations of 26 faint $(g \geq 18.5)$ WDMS binaries, which led to identifying 9 new PCEBs through radial velocity variations. The orbital periods of two of these PCEBs were determined from additional Magellan-Clay/LDSS3 observations. The structure of the paper is as follows. In Sect. 2 we describe the selection of the 26 VLT targets and the determination of their stellar parameters. Details of the observations and data reduction of the VLT and Magellan-Clay runs are given in Sect. 3. After describing our method of measuring radial velocities and determining orbital periods (Sect. 4), we discuss our results in the context of our large-scale PCEB survey.

\section{Target selection and stellar parameters}

For this project, we selected 26 WDMS binaries with $i<19.5$ and visible from Paranal from the SDSS Data release 5 (DR5, Adelman-McCarthy et al. 2007), using the lists compiled by Eisenstein et al. (2006), Silvestri et al. (2007) and Southworth et al. (2007). Given the recently suggested test of disrupted magnetic braking (Politano \& Weiler 2006), we focussed this selection on WDMS binaries with intermediate M-type (M2-M5) companions. Table 1 lists the SDSS object names and the $i$ magnitudes of our targets.

We have determined the stellar parameters of these 26 WDMS binaries following the method described in Paper I, with only the difference of now using spectra retrieved from Data Release 6, which have undergone a complete re-reduction that substantially improves the flux calibration (Adelman-McCarthy et al. 2008). For the sake of brevity, we provide here only a brief summary of the adopted procedures, but the reader is referred to Paper I for full details. We first carry out a two-component fit to the SDSS WDMS spectra using libraries of observed white dwarf and M-dwarf template spectra, normalised to stellar surface fluxes. These template libraries are also compiled from SDSS DR6. This stage provides us with a spectral type for the companion star and a flux scaling factor for the M-dwarf template that, together with a spectral type-radius relation, yields a distance estimate. In a second step, we subtract the best-fit M-dwarf template from the WDMS spectra and fit the residual white dwarf spectra with a standard $\chi^{2}$ method for variable white dwarf effective temperature $\left(T_{\text {eff }}\right)$ and surface gravity $(\log g)$, using a grid of pure-hydrogen (DA) model spectra computed with the code of Koester et al. (2005) ${ }^{1}$. Two flavours of white dwarf fits were carried out for each object. (1) First there's a fit to the normalised line profiles. Due to the degeneracy in Balmer line equivalent width as a function of temperature, two acceptable fits are usually found, a "hot" and a "cold" solution. (2) There is also a fit to the continuum plus lines over the range 3850-7150 $\AA$. The parameters from this fit are subject to substantial systematic uncertainties due to inaccuracies in the spectral slope, and we only use them to break the degeneracy between the "hot" and "cold" line profile solutions. Examples of the spectral decomposition and of the white dwarf fits are shown in Figs. 1 and 2.

\footnotetext{
1 We limit the spectral fitting to DA white dwarfs, but provide a spectral type of the companion for the two helium atmosphere (DB) WDMS binary in our sample.
} 

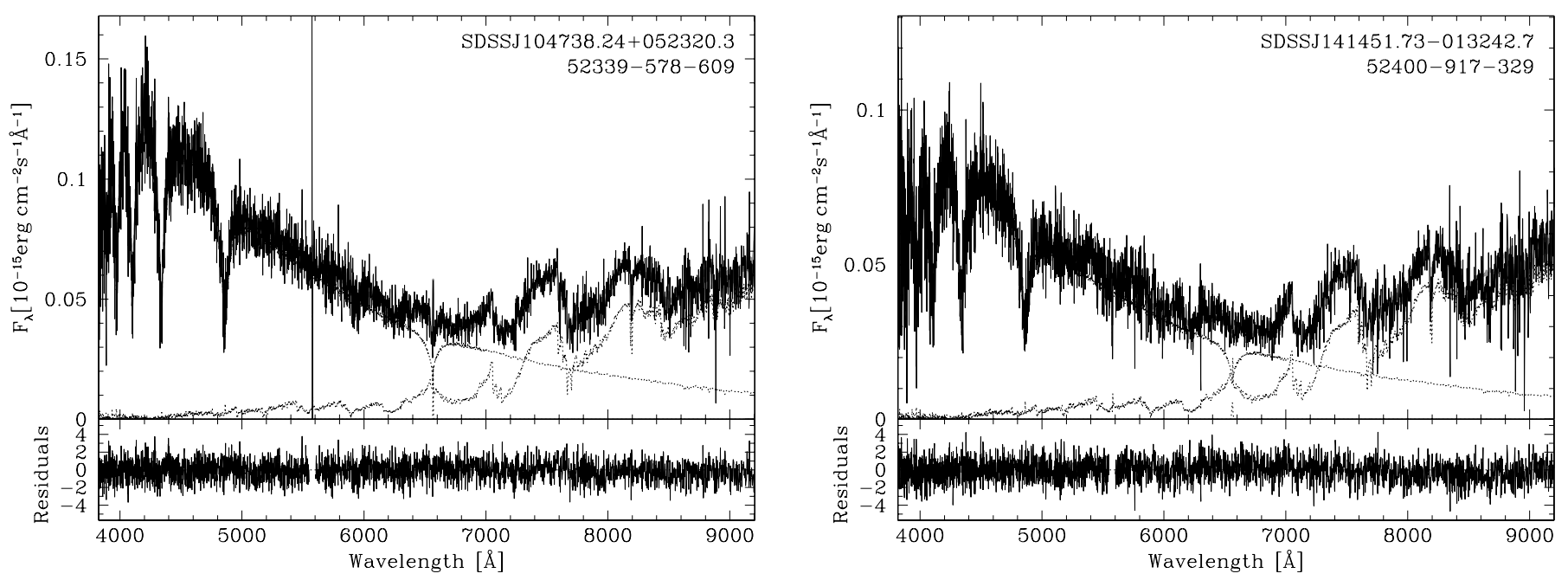

Fig. 1. Two-component fits to the 2 PCEBs SDSS J1047+0523 and SDSS J1414-0132, for which we determined orbital periods from Clay/LDSS3 spectroscopy. Top panels: the WDMS spectra (black lines) along with the white dwarf and M-dwarf templates (dotted lines). The MJD-PLT-FIB identifiers of the two SDSS spectra are also given. Bottom panels: residuals from the fit.
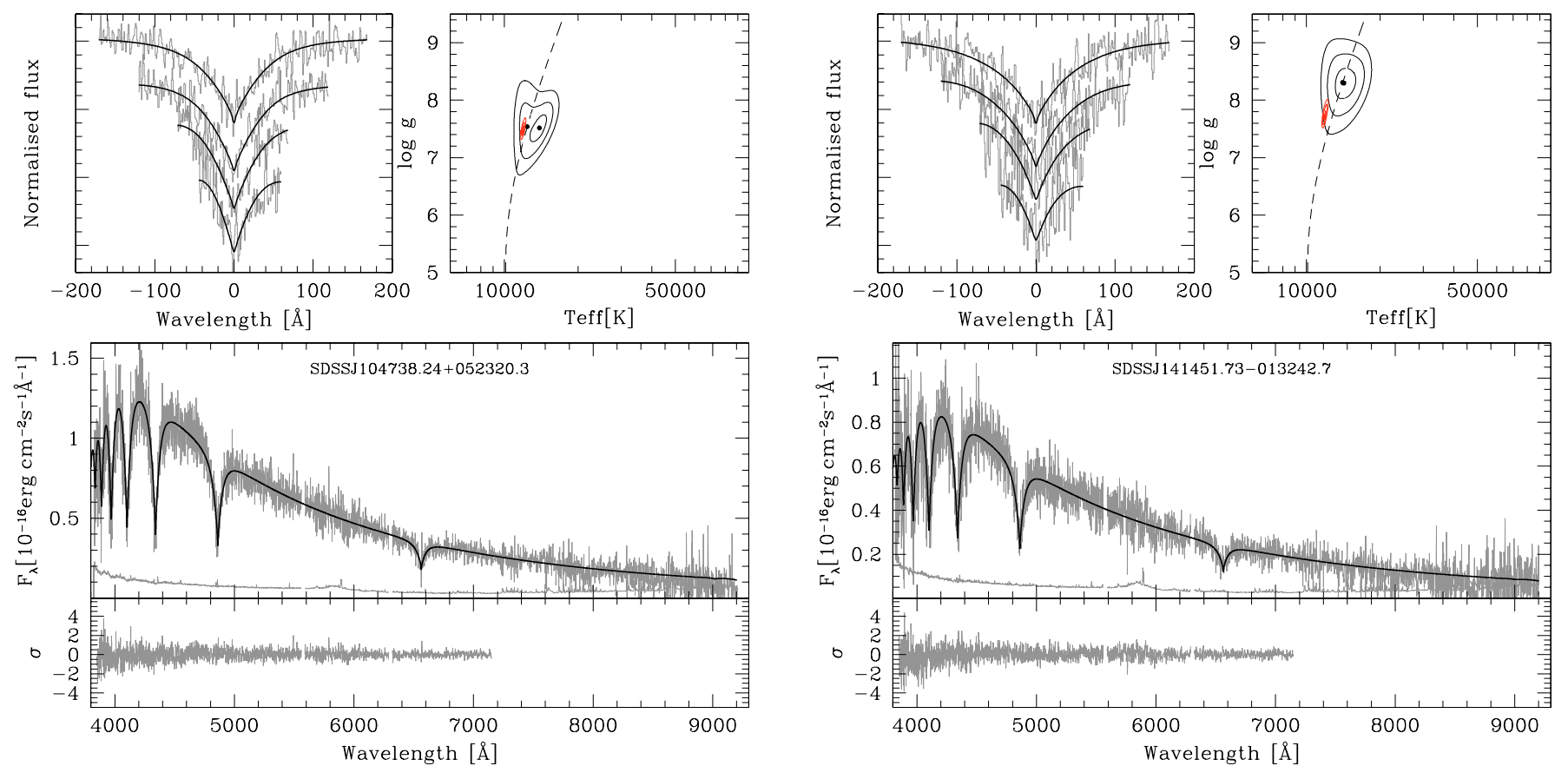

Fig. 2. Spectral model fits to the white dwarf components of SDSS J1047+0523 and SDSS J1414-0132, obtained by subtracting the best-fit Mdwarf template from their SDSS spectra. Top left panels: normalised $\mathrm{H} \beta$ to $\mathrm{H} \epsilon$ line profiles (top to bottom, gray lines) along with the best-fit white dwarf model (black lines). Top right panels: 1, 2, and $3 \sigma \chi^{2}$ contour plots in the $T_{\text {eff }}-\log g$ plane. The black contours refer to the best line profile fit, the red contours to the fit of the whole spectrum. The dashed line indicates the location of maximum $\mathrm{H} \beta$ equivalent width in the $T_{\text {eff }}-\log g$ plane, dividing it into "hot" and "cold" solutions. The best-fit parameters of the "hot" and "cold" normalised line profile solutions and of the fit to the 3850-7150 ̊ range are indicated by black and red dots, respectively. Bottom panels: the white dwarf spectrum and associated flux errors (gray lines) along with the best-fit white dwarf model (black lines) to the 3850-7150 ̊ wavelength range (top) and the residuals of the fit (gray line, bottom).

We then used an updated version of the tables in Bergeron et al. $(1995)^{2}$, which incorporate the structure and evolution calculations of Wood (1995) and Fontaine et al. (2001), to compute white dwarf masses and radii from $T_{\text {eff }}$ and $\log g$. Finally, the white dwarf radii and flux scaling factors of the white dwarf models yield a second distance estimate. Statistical errors on

\footnotetext{
${ }^{2}$ http://www.astro.umontreal.ca/ bergeron/ CoolingModels/
}

the different properties are propagated through this process, but should be considered with some caution as additional systematic uncertainties are not easy to quantify. The resulting stellar parameters are listed in Table 1. Inspection of Fig. 3 (left panel) shows, that in about one third of the systems, the distance estimate based on the fit of the secondary star exceeds the estimate based on the white dwarf fit by a significant $(>1 \sigma)$ factor. A similar ratio of systems with $d_{\mathrm{sec}}>d_{\mathrm{wd}}$ has been found in the much larger sample analysed in PaperI, which also discusses 

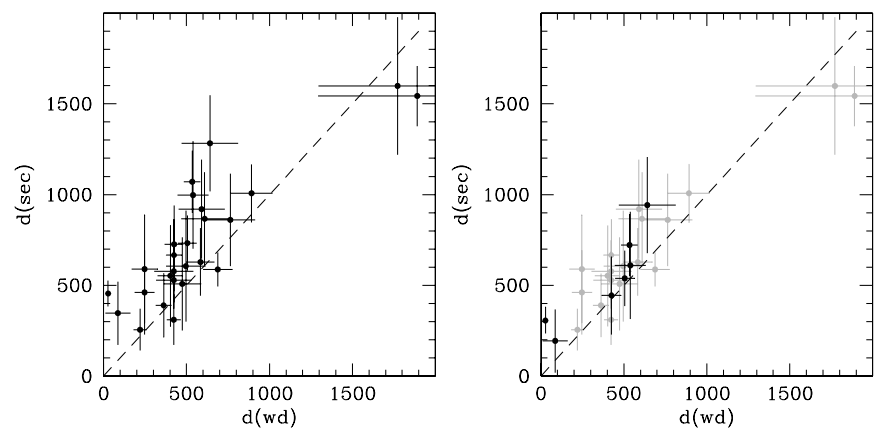

Fig. 3. Distances of the 24 WDMS binaries in Table 1 that contain a DA white dwarf. The spectral modelling (Sect. 2) provides two independent distance estimates for the individual stellar components. Nine systems have $d_{\mathrm{sec}}>d_{\mathrm{wd}}$ by more than $1 \sigma$. Adopting secondary star spectral types later by one class brings most of them onto the $d_{\mathrm{sec}}=d_{\mathrm{wd}}$ relation. An earlier appearance of the spectral type determined from an optica spectrum may be related to stellar activity. See Paper I for additional discussion.

possible explanations for this finding in detail. Our favoured hypothesis from Paper I is that stellar activity causes large scatter in the spectral type-radius relation of low-mass stars (in fact, more than the scatter in their mass-radius relation that is subject to thorough investigations, e.g. Ribas 2006; López-Morales 2007), with a tendency toward spectral types that are too early for their radius. In the right hand panel of Fig. 3, we show that adopting secondary stars of one spectral class later than in Table 1 brings the nine systems that had $d_{\mathrm{sec}}>d_{\mathrm{wd}}$ close to the $d_{\mathrm{sec}}=d_{\mathrm{wd}}$ relation.

A final note concerns SDSS J2324-0931, which contains a DB white dwarf. The DR3 SDSS spectrum of SDSS J2324-0931 was analysed by van den Besselaar et al. (2005) who adopted a method similar to our spectral decomposition, i.e. fitting the WDMS spectrum with the combination of observed white dwarf and M-dwarf templates. Unlike our approach, they fixed the system distance to be the same for both stars. Their analysis resulted in $T_{\text {eff }}=36815, \mathrm{Sp}(2)=\mathrm{M} 3 \mathrm{~V}$, and $d=911 \mathrm{pc}$, while our spectral decomposition results in $\mathrm{Sp}(2)=\mathrm{M} 4 \mathrm{~V}$ and $d=633 \pm 188 \mathrm{pc}$. By Forcing the spectral type to M3V, we obtain a distance of $879 \pm 140$ pc. Given that the typical uncertainty in the spectral type is one spectral class, the two results are consistent.

\section{Observations}

\subsection{VLT/FORS2}

Intermediate resolution spectroscopy of 26 WDMS binaries were obtained between August 16, 2007 and March 26, 2007 with FORS2 on the VLT/UT1 (Antu). The exposure time was $900 \mathrm{sec}$ for all objects. The observations were carried out using the $1028 \mathrm{z}$ grism and a 1 " slit, resulting in a spectral coverage of 7830-9570 . The images were processed using the STARLINK packages FIGARO and KAPPA, and the spectra were optimally extracted (Horne 1986) using the PAMELA package (Marsh 1989). From measurements of the FWHMs of sky lines, we find our observations have a resolution of approximately 2.5 pixels $(2.2 \AA)$ at $8200 \AA$. Wavelength calibration of the extracted spectra was done using only sky emission lines. The wavelengths of good sky lines were obtained from the atlas of Osterbrock et al. (1996, 1997). Thirty-seven sky lines were included, and fitted with a fifth-order polynomial. The rms scatter around the fit was $0.11 \AA$, so the statistical uncertainty in the wavelength scale is $0.04 \AA$
(Marsh et al. 1994). Finally, the spectra were flux-calibrated and compensated for telluric absorption using a spectrum of the standard star Feige 110.

The Phase II design of these observations was to obtain two spectra per object, separated by at least one night, in order to probe for radial velocity variations. Due to poor atmospheric conditions, additional repeat observations were obtained for several targets, while two objects only got one FORS2 spectrum each.

\subsection{Magellan-Clay/LDSS3}

Follow-up spectroscopy of two PCEBs identified from the FORS2 data, SDSS J1047+0523 and SDSS J1414-0123, were obtained over the period 2007 May, 17-20 using the Magellan Clay telescope equipped with the LDSS3 imaging spectrograph. Seeing and transparency were highly variable. We adjusted the exposure times accordingly, ranging from 500-900 s for SDSS J1047+0523 and 750-1200s for SDSS J1414-0123. We used the VPH_Red grism and OG 590 order-sorting filter. The detector was an unbinned STA $4 \mathrm{k} \times 4 \mathrm{k}$ pixel CCD read out by two amplifiers. A $0.75^{\prime \prime}$ offset slit gave a wavelength coverage of 5800-9980 $\AA$ at a reciprocal dispersion of $1.2 \AA \mathrm{px}^{-1}$. We took flat-field images at each sky position whilst observing, as this was needed to remove the effects of fringing at redder wavelengths. The dispersed images were processed using the same software as for the VLT observations. Measurements of the FWHMs of the sky lines indicate that our observational setup gave a resolution of approximately 4 pixels $(4.8 \AA)$ at $8200 \AA$. Wavelength calibration was done using only sky lines to increase observational efficiency. A total of 36 sky lines were fitted by a fifth-order polynomial. The rms scatter around the fit was $0.25 \AA$, so the statistical uncertainty is $0.09 \AA$. From this work and other studies we have found that reliable wavelength calibrations can be obtained from sky lines (see also Southworth et al. 2006), but that the scatter of the fit around the wavelength solution is quite a bit larger than for arc-lamp emission lines. In this case the statistical uncertainty is equivalent to $0.09 \AA$ so the increased scatter is relatively unimportant.

Flux calibration and telluric line removal were performed using spectra of the standard star LTT 3218 obtained during the same observing run.

\section{Radial velocity variations and orbital periods}

We used the strong Na I $\lambda \lambda 8183.27,8194.81$ absorption doublet present in the spectra of all our targets to measure the radial velocities of the main-sequence companion stars. For this purpose, we fitted the wavelength of the Na I doublet using the sum of a linear slope for the continuum plus two Gaussians for the absorption lines. We fixed the separation of the two Gaussians to $11.54 \AA$ corresponding to the laboratory value. Free parameters in the fit were the continuum flux level and slope, the wavelength of the doublet, the FWHM of the lines (both FWHM were fixed to vary together), and the amplitudes of the individual doublet components. The fits were carried out using the fit/table command in MIDAS. Examples of these fits are shown in Fig. 5. We added the uncertainty in the wavelength calibration in quadrature to the statistical errors in the wavelength of the Na I resulting from the double-Gaussian fit.

One system, SDSS J1222+0934, has an M0V secondary star, which exhibits no significant $\mathrm{Na}$ I absorption. For this single object, we used the same method as above, but fitted three 
Table 1. Stellar parameter of the 26 WDMS binaries observed with FORS2, as determined from spectral modelling (Sect. 2).

\begin{tabular}{|c|c|c|c|c|c|c|c|c|c|}
\hline System (SDSS J) & MJD-plate-fibre & $i$ & $T[\mathrm{~K}]$ & $\log g$ & $M_{\mathrm{wd}}\left[M_{\odot}\right]$ & $d_{\mathrm{wd}}[\mathrm{pc}]$ & $\mathrm{Sp}$ & $d_{\mathrm{sec}}[\mathrm{pc}]$ & notes \\
\hline $001726.63-002451.1$ & $52559-1118-280$ & 18.3 & $12828 \pm 2543$ & $00 \pm 0.46$ & $0.61 \pm 0.29$ & $423 \pm 119$ & 4 & $577 \pm 171$ & \\
\hline - & $52518-0687-153$ & 18.3 & $13588 \pm 1803$ & $8.11 \pm 0.39$ & $0.68 \pm 0.25$ & $423 \pm 107$ & 4 & 529 & \\
\hline $022835.92-074032.3$ & 51908-0454-534 & 18.7 & $13588 \pm 948$ & $7.76 \pm 0.17$ & $0.48 \pm 0.09$ & $423 \pm 43$ & 6 & $311 \pm 140$ & \\
\hline $032038.72-063822.9$ & 51924-0460-432 & 18.7 & $11045 \pm 479$ & $8.49 \pm 0.33$ & $0.91 \pm 0.19$ & $247 \pm 61$ & 5 & $461 \pm 232$ & $1, P_{\text {orb }} \leq$ \\
\hline $081959.20+060424.2$ & $52962-1296-138$ & 19.2 & $15782 \pm 1422$ & $7.67 \pm 0.34$ & $0.45 \pm 0.17$ & $764 \pm 150$ & 4 & $860 \pm 255$ & \\
\hline $083354.84+070240.1$ & $52963-1297-511$ & 18.5 & $16336 \pm 730$ & $8.03 \pm 0.16$ & $0.63 \pm 0.10$ & $424 \pm 45$ & 4 & $666 \pm 198$ & $\leq 14.7 \mathrm{~d}$ \\
\hline $085542.49+044717.7$ & $52670-1190-512$ & 19.1 & $10073 \pm 336$ & $8.46 \pm 0.43$ & $0.89 \pm 0.25$ & $247 \pm 77$ & 5 & $589 \pm 297$ & $1.9 \mathrm{~d}$ \\
\hline 092451.63-001736.4 & 52000-0474-175 & 17.6 & $24163 \pm 1359$ & $7.82 \pm 0.20$ & $0.54 \pm 0.11$ & $688 \pm 89$ & 3 & $588 \pm 94$ & \\
\hline $093904.03+051114.8$ & 52710-0993-296 & 19.2 & $12681 \pm 1406$ & $7.69 \pm 0.42$ & $0.44 \pm 0.23$ & $591 \pm 139$ & 4 & $919 \pm 273$ & \\
\hline $095108.74+025507.5$ & 51908-0481-419 & 18.5 & $13432 \pm 1007$ & $7.80 \pm 0.22$ & $0.50 \pm 0.12$ & $425 \pm 57$ & 4 & $726 \pm 215$ & \\
\hline 100953.69-002853.4 & $51909-0$ & 19.0 & $21535 \pm$ & $8.35 \pm$ & $0.84 \pm 0.19$ & 141 & 4 & 867 & \\
\hline $104358.59+060320.9$ & $00-055$ & 18.6 & 536 & $8.39 \pm$ & $0.85=$ & & 6 & 256 & \\
\hline $104738.24+05$ & -609 & 18.7 & 123 & $7.54 \pm$ & $0.38 \pm 0.20$ & 11 & 5 & 508 & $1,2, P_{\text {orb }}<0.8 \mathrm{~d}$ \\
\hline $110151.34+122241.5$ & 531 & 18.8 & 817 & $7.83=$ & 0.5 & & 3 & 171 & \\
\hline $112623.87+010856.8$ & 516 & 17.5 & 1 & $1 \pm$ & 0.5 & 50 & 2 & 73 & \\
\hline $122208.48+093406.2$ & $52672-1230-73$ & 18.3 & $30419 \pm 2053$ & $7.34 \pm 0.49$ & $0.38 \pm 0.18$ & 1891 & 0 & 166 & 4 \\
\hline $125250.03-020608.1$ & 51694-0338-343 & 18.5 & $22037 \pm 2333$ & $8.29 \pm 0.38$ & $0.80 \pm 0.23$ & $641 \pm 171$ & 2 & $1282 \pm 265$ & \\
\hline $141451.73-013242.7$ & 52400-0917-329 & 19.0 & $14065 \pm 1452$ & $8.31 \pm 0.27$ & $0.80 \pm 0.17$ & $402 \pm 77$ & 5 & $552 \pm 278$ & $1,3,6, P_{\text {orb }}<3.4 \mathrm{~d}$ \\
\hline $142541.32+023047.4$ & 51999-0535-348 & 18.9 & $13127 \pm 1014$ & $7.91 \pm 0.22$ & $0.56 \pm 0.13$ & $362 \pm$ & 6 & $390 \pm 176$ & \\
\hline $150657.58-012021.7$ & $52426-0922-536$ & 18.4 & $15782 \pm 1005$ & $7.72 \pm 0.24$ & $0.47 \pm 0.13$ & $584 \pm$ & 4 & $629 \pm 187$ & \\
\hline $204541.90-050925.7$ & $52145-0635-439$ & 18.7 & $26801 \pm 1432$ & $7.78 \pm 0.22$ & $0.53 \pm 0.11$ & $892 \pm 127$ & 3 & $1007 \pm 160$ & , $P_{\text {orb }}<49.1 \mathrm{~d}$ \\
\hline 205059.37-000254.3 & 52466-0982-567 & 19.2 & $18330 \pm 1169$ & $8.27 \pm 0.24$ & $0.78 \pm 0.15$ & $539 \pm 93$ & 4 & $998 \pm 296$ & \\
\hline $212320.74+002455.5$ & 52523-0987-484 & 19.2 & $12250 \pm 1794$ & $7.47 \pm 0.42$ & $0.35 \pm 0.19$ & $496 \pm 119$ & 5 & $606 \pm 305$ & \\
\hline 215614.57-000237.4 & 52078-0371-025 & 18.5 & $7910 \pm 926$ & $9.50 \pm 0.74$ & $1.37 \pm 0.21$ & $86 \pm 76$ & 5 & $346 \pm 174$ & $1,4,6, P_{\text {orb }}<44.0 \mathrm{~d}$ \\
\hline $220338.61-001750.7$ & 52173-0372-034 & 18.7 & $32972 \pm 1703$ & $7.24 \pm 0.38$ & $0.37 \pm 0.11$ & $1772 \pm 477$ & 1 & $1598 \pm 379$ & \\
\hline $231221.59+010127.0$ & 51811-0381-570 & 17.3 & - & - & - & - & 3 & $455 \pm 72$ & 5 \\
\hline $232438.31-093106.4$ & 52203-0645-637 & 18.4 & - & - & - & - & 4 & $633 \pm 188$ & 5 \\
\hline
\end{tabular}

(1) PCEB candidate on the base of radial velocity variations detected among the available FORS2 and SDSS spectra; (2) $P_{\text {orb }}=9.17 \mathrm{~h}$ determined from Magellan-Clay/LDSS3 spectroscopy; (3) $P_{\text {orb }}=17.48 \mathrm{~h}$ determined from Magellan-Clay/LDSS3 spectroscopy; (4) blue component is faint, white dwarf parameter uncertain; (5) DB white dwarf; (6) poor quality spectrum. Upper limits on the orbital periods for the nine PCEBs identified by our VLT/FORS2 observations are calculated as described in Sect. 5.1.

Gaussian lines of fixed separation and common width to the Ca II $\lambda \lambda$ 8498.02, 8542.09, 8662.14 triplet.

We combined the radial velocities obtained from the FORS2 data with those measured from the DR6 SDSS spectra (Table 3). Comparing the individual radial velocities of each object, we define as strong PCEB candidates all systems that show a radial velocity variation at $\mathrm{a} \geq 3 \sigma$ level. Applying this criterion, we identified 9 PCEB candidates among our target sample of 26 WDMS binaries (Fig. 4, Table 1).

The radial velocity variations of the companion stars in SDSS J1414-0132 and SDSS J1047+0523 were determined in an analogous fashion to what is described above from the Clay/LDSS3 spectroscopy. We then calculated Scargle (1982) periodograms from the mean-subtracted radial velocity measurements to establish the periods of the two WDMS binaries (Fig. 6). We carried out sine-fits to the radial velocities using the three highest aliases in the periodograms as initial value for the frequency, and report the resulting periods, $\gamma$-velocities and radial velocity amplitudes, as well as their errors and the reduced $\chi^{2}$ values, in Table 2 .

Both the lowest reduced $\chi^{2}$ values and the lowest relative errors on the fit parameters are found for the periods corresponding to the highest peaks in the periodograms. We therefore conclude that the orbital periods of SDSS J1414-0132 and SDSS J1047+0523 are $P_{\text {orb }}=17.48 \pm 0.07 \mathrm{~h}$ and $P_{\text {orb }}=$ $9.17 \pm 0.02 \mathrm{~h}$. Folding the radial velocities over the neighbouring aliases results in substantially distorted non-sinusoidal periods. The LDSS3 radial velocities of SDSS J1414-0132 and SDSS J1047+0523 folded over their orbital periods are shown
Table 2. Orbital periods, semi-amplitudes, and reduced $\chi^{2}$ from sinefits to the radial velocity data of SDSS J1047+0523 and SDSS J14140132 for the strongest three aliases in the periodograms shown in Fig. 6. The best-fit values are set in bold font.

\begin{tabular}{lrrrr}
\hline \hline System & $P_{\text {orb }}[\mathrm{h}]$ & $K_{2}\left[\mathrm{~km} \mathrm{~s}^{-1}\right]$ & $\gamma\left[\mathrm{km} \mathrm{s}^{-1}\right]$ & $\chi^{2}$ \\
\hline SDSS J1047+0523 & $15.11 \pm 0.12$ & $195 \pm 16$ & $20 \pm 11$ & 14.4 \\
& $\mathbf{9 . 1 7} \pm \mathbf{0 . 0 2}$ & $\mathbf{1 8 7} \pm \mathbf{7}$ & $\mathbf{4 5} \pm \mathbf{5}$ & $\mathbf{2 . 8}$ \\
& $6.64 \pm 0.04$ & $181 \pm 22$ & $66 \pm 17$ & 27.6 \\
SDSS J1414-0132 & $51.01 \pm 0.53$ & $371 \pm 32$ & $44 \pm 12$ & 10.8 \\
& $\mathbf{1 7 . 4 8} \pm \mathbf{0 . 0 7}$ & $\mathbf{1 6 8} \pm \mathbf{4}$ & $\mathbf{3 4} \pm \mathbf{3}$ & $\mathbf{1 . 0}$ \\
& $10.14 \pm 0.09$ & $152 \pm 13$ & $46 \pm 10$ & 13.0 \\
\hline
\end{tabular}

in Fig. 7. Below we provide some comments on individual objects.

SDSS J0017-0024: Two SDSS spectra are available for this object, with radial velocities that are consistent at a $2 \sigma$ level (Rebassa-Mansergas et al. 2007). We obtained two FORS2 spectra, which agree within their $1 \sigma$ errors, confirming this system as a likely wide binary WDMS.

SDSS J1047+0523: Only a single VLT/FORS2 spectrum was obtained for this object. However, combining the radial velocity 


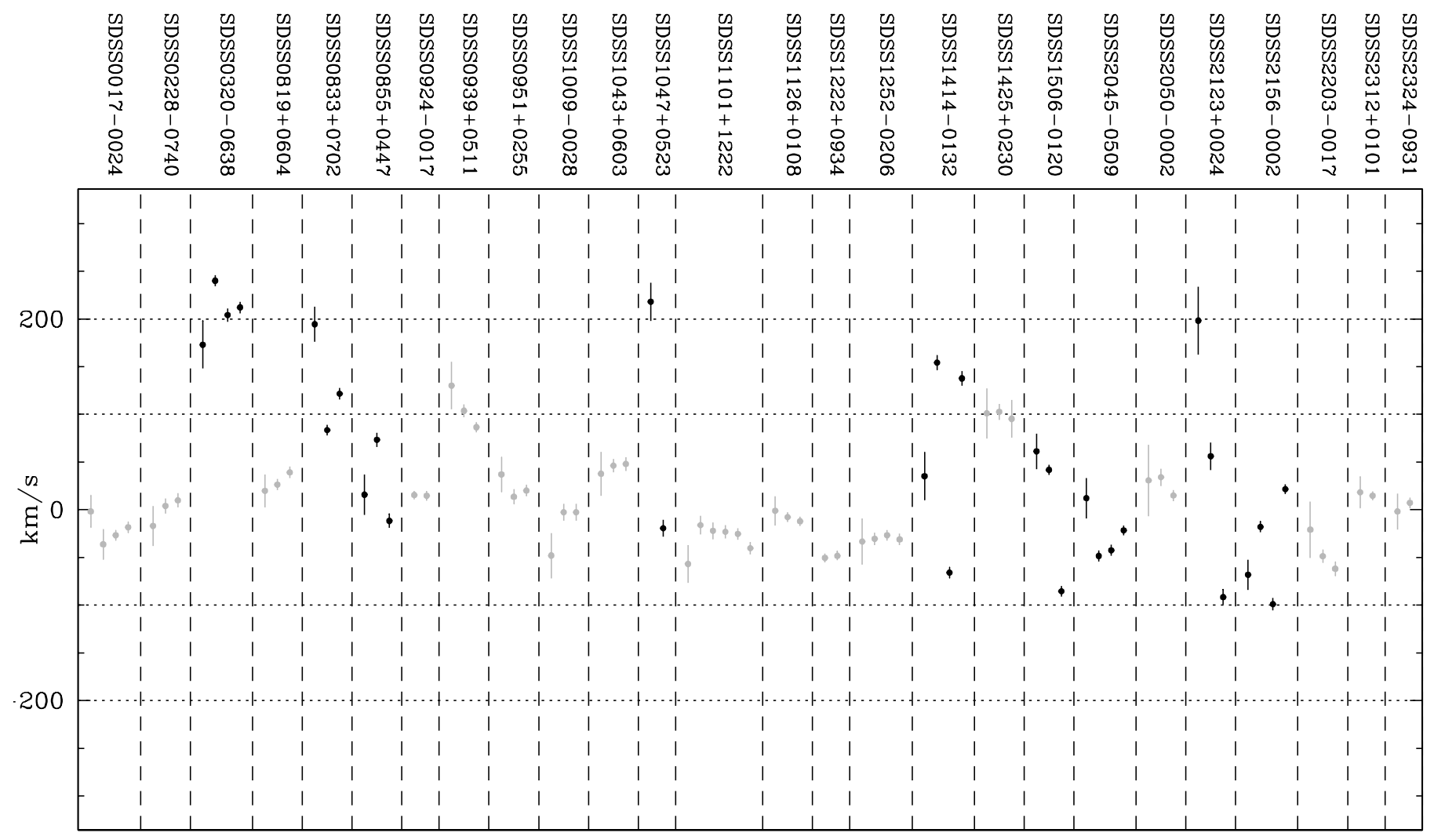

Fig. 4. Radial velocities (Table 3) of our sample 26 WDMS binaries (Table 1), measured from the Na I $\lambda \lambda 8183.27,8194.81$ doublets observed in their SDSS and FORS2 spectra. No SDSS radial velocities could be determined for SDSS J1252-0206, SDSS J1414-0132, and SDSS J2050-0002. See Fig. 5 for examples of the Na I $\lambda \lambda 8183.27,8194.81$ fits. The systems with black symbols display radial velocity variations at a $3 \sigma$ level.

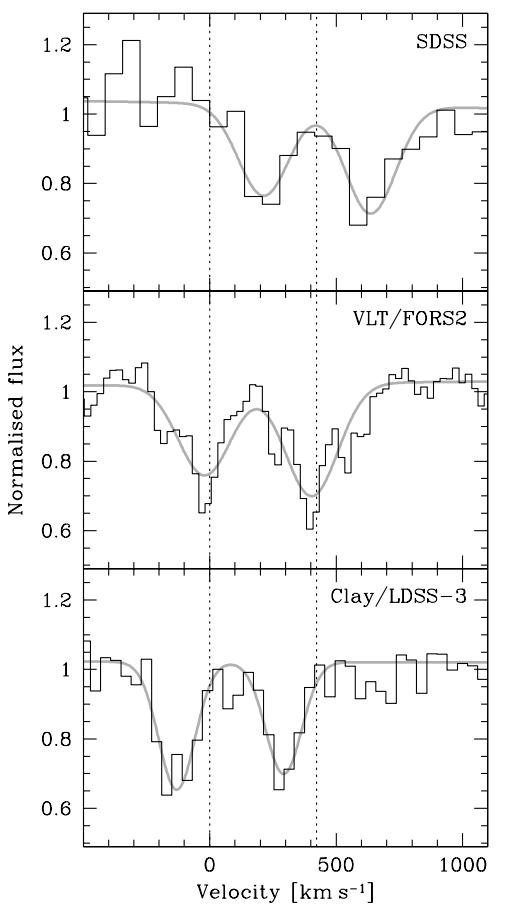

Fig. 5. Examples of the double-Gaussian fits to the NaI $\lambda \lambda 8183.27,8194.81$ line profiles in the SDSS, VLT/FORS2, and Clay/LDSS3 spectra of SDSS J1047+0523.

from the FORS2 spectrum with the one measured from the SDSS spectrum clearly identifies the system as a close binary.

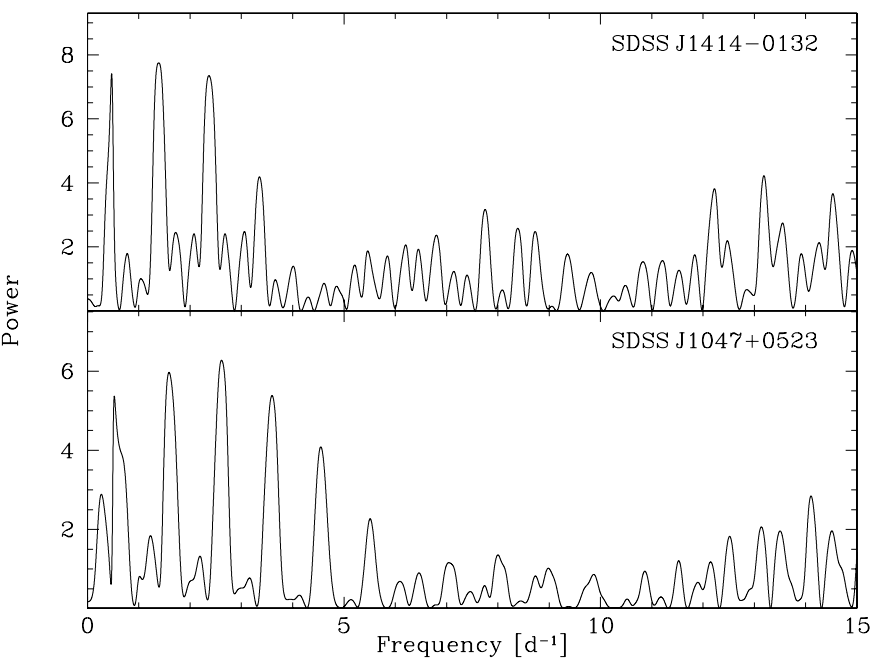

Fig. 6. Scargle periodograms calculated from the Na I $\lambda \lambda 8183.27,819-$ 4.81 radial velocities measured in the LDSS3 spectra of SDSS J14140123 and SDSS J1047+0523.

\section{Discussion}

\subsection{Orbital period limits}

As in Rebassa-Mansergas et al. (2007), we determine an upper limit for the orbital period based on the measured radial velocity variations (Table 3). The orbital period as a function of the stellar 


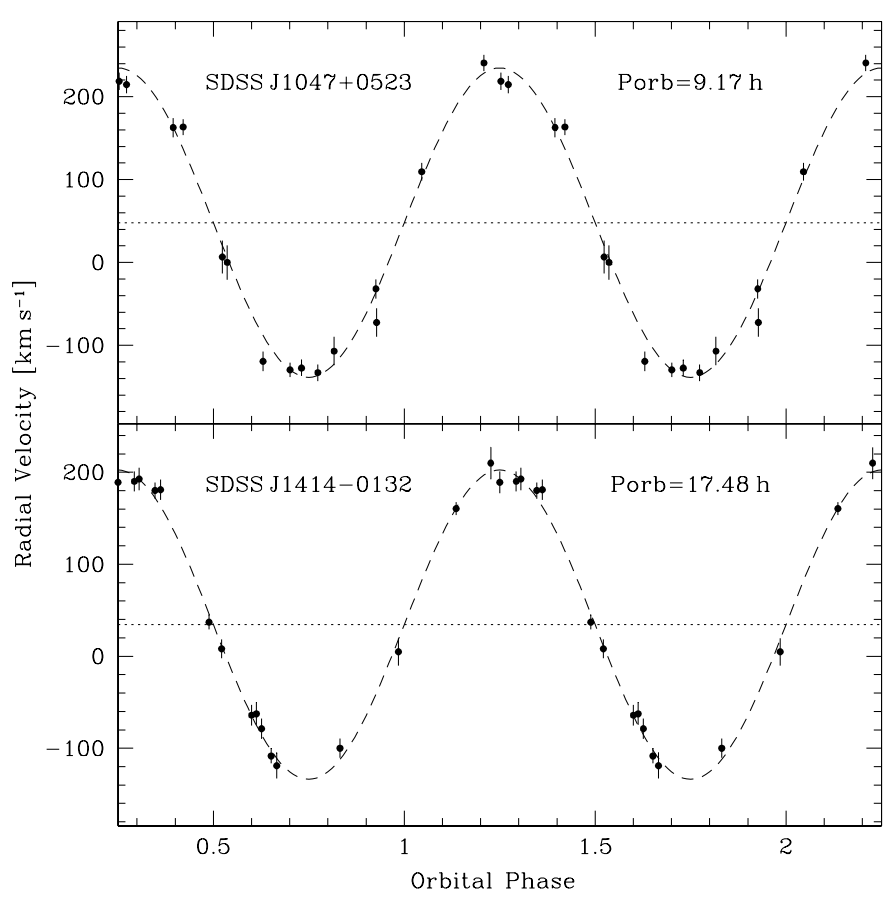

Fig. 7. The Na I $\lambda \lambda 8183.27,8194.81$ radial velocities of the companion stars in SDSS J1414-0132 and SDSS J1047+0523 folded over their respective orbital periods of $P_{\text {orb }}=17.48 \mathrm{~h}$ and $P_{\text {orb }}=9.17 \mathrm{~h}$.

masses, the binary inclination $(i)$, and the radial velocity amplitude $\left(K_{\mathrm{sec}}\right)$ of the secondary follows from Kepler's 3rd law:

$\frac{\left(M_{\mathrm{wd}} \sin i\right)^{3}}{\left(M_{\mathrm{wd}}+M_{\mathrm{sec}}\right)^{2}}=\frac{P_{\mathrm{orb}} K_{\mathrm{sec}}^{3}}{2 \pi G}$.

Using the stellar parameters estimated in Sect. 2 from fitting the SDSS spectrum, assuming $i=90^{\circ}$, and assuming the largest radial velocity difference measured for one particular system to be equal to $2 K_{\mathrm{sec}}$ clearly gives an upper limit on $P_{\text {orb }}$. This method was used in Rebassa-Mansergas et al. (2007). Here we slightly improve this approach by using also the information provided by the times of observations. If the time delay between two spectra $(\Delta t)$ is shorter than half the considered orbital period, the maximal radial velocity difference that could have been observed writes as $2 K_{\mathrm{sec}} \sin \left(\pi \Delta t / 2 P_{\text {orb }}\right)$, and we modify Eq. (1) accordingly. Upper limits on the orbital period for the 9 PCEBs obtained with this method are given in Table 1.

\subsection{The fraction of PCEBs among SDSS WDMS binaries}

The first result of our PCEB survey will be the relative number of PCEBs among SDSS WDMS binaries. According to the derived limits on the orbital periods and the masses given in Table 1, the 9 close WDMS binaries identified in this paper have clearly experienced a phase of mass transfer during the post main sequence evolution of the progenitor of the white dwarf. As mentioned in the introduction, according to current WDMS binary population studies (with many uncertainties involved), the majority of all WDMS that formed through mass transfer interactions are expected to be PCEBs, and only a small fraction of close systems may form through dynamically stable mass transfer. For the 9 PCEB candidates identified among our 26 WDMS systems dynamically stable mass transfer appears to be extremely unlikely as the white dwarf masses are rather high and the secondary
Table 3. Radial velocities measured from the Na I $\lambda \lambda 8183.27,8194.81$ doublet in the ESO/VLT and SDSS spectra. The radial velocities obtained from SDSS data is indicated by the HJD set in italics.

\begin{tabular}{|c|c|c|c|}
\hline HJD & $R V\left[\mathrm{~km} \mathrm{~s}^{-1}\right]$ & HJD & $R V\left[\mathrm{~km} \mathrm{~s}^{-1}\right]$ \\
\hline \multicolumn{2}{|c|}{ SDSS J0017-0024 } & \multicolumn{2}{|c|}{ SDSS J1126+0108 } \\
\hline 2453978.64799 & $-26.7 \pm 5.0$ & 2454142.80234 & $-7.7 \pm$ \\
\hline 82.6 & -1 & 454155.62142 & -12.1 \\
\hline 922000 & 16.7 & 451614.80814 & $-1.1 \pm$ \\
\hline 2452559.785000 & $-36.3 \pm 15.5$ & \multicolumn{2}{|c|}{ SDSS J1222+0934 } \\
\hline \multicolumn{2}{|c|}{ SDSS J0228-0740 } & 142.831916 & $-50.4=$ \\
\hline 83.68162 & $40+-4$ & 156.875771 & \\
\hline 2453983.69278 & 5 & \multicolumn{2}{|c|}{ SDSS J1252-0206 } \\
\hline - & - & 4647 & 304 \\
\hline \multicolumn{2}{|c|}{ SDSS J0320-0638 } & & \\
\hline 30.8 & & & \\
\hline 24 & & 694.70928 & \\
\hline 24. & & \multicolumn{2}{|c|}{ SDSS J1414-0132 } \\
\hline $924.0 / 640$ & 172 & 245414 & 1.3 \\
\hline \multicolumn{2}{|c|}{ SDSS J0819+0604 } & 1 & \\
\hline 2454030.87927 & .7 & 24 & \\
\hline 24 & .0 & & $5 J .2$ \\
\hline 2452963.00286 & $19.8 \pm 17.1$ & \multicolumn{2}{|c|}{ SDSS J1425+0230 } \\
\hline \multicolumn{2}{|c|}{ SDSS J0833+0702 } & 245398 & $102.6 \pm 8.4$ \\
\hline 245 & $83.5 \pm 5.5$ & 22 & \\
\hline & & 2451999.89365 & 101.1 \\
\hline 436 & $194.5 \pm$ & \multicolumn{2}{|c|}{ SDSS J1506-0120 } \\
\hline \multicolumn{2}{|c|}{ SDSS J0855+0447 } & & \\
\hline & & & \\
\hline & & 2452426 & \\
\hline 24 & & \multicolumn{2}{|c|}{ SDSS J2045-0509 } \\
\hline \multicolumn{2}{|c|}{ SDSS J0924-0017 } & 49 & -48.4 \\
\hline 67.8 & & & \\
\hline J0 & & P & \\
\hline SDS & $9+0$ & 2452145.71287 & $12.1 \pm 21.2$ \\
\hline 086.81276 & 103 & \multicolumn{2}{|c|}{ SDSS J2050-0002 } \\
\hline 7.84233 & 4 & 3998.625 & $34.1 \pm 9.1$ \\
\hline 710.81094 & 130 & 00 & 5.8 \\
\hline \multicolumn{2}{|c|}{ SDSS J0951+0255 } & 466. & $30.8 \pm 37.5$ \\
\hline 86.8 & 7.4 & \multicolumn{2}{|c|}{ SDSS J2123+0024 } \\
\hline 24 & 6 & 978 & $56.1 \pm$ \\
\hline 08.91909 & 37.0 & 981 & \\
\hline \multicolumn{2}{|c|}{ SDSS J1009-0028 } & 331 & 198.2 \\
\hline & & \multicolumn{2}{|c|}{ SDSS J2156-0002 } \\
\hline & & & \\
\hline 24 & -48 . & & \\
\hline SDSS J10 & $43+0603$ & 6 & 5.1 \\
\hline 117.75282 & $162+$ & 2452075.89855 & -68 . \\
\hline 149.70110 & 2 & SDSS J22 & 3-0 \\
\hline 2452644.01881 & $37.7 \pm 22.9$ & 2453978.57300 & $-48.7 \pm 6.4$ \\
\hline SDSS J10 & $47+0$ & 3714 & -61.9 \\
\hline 142.77188 & $-19.4 \pm 8.9$ & 2452173.63031 & $-20.9 \pm 29.5$ \\
\hline 2452339.8 & $218.0 \pm 19.8$ & SD & $2+01$ \\
\hline SD & & 978 & 14 . $^{\circ}$ \\
\hline 142.78686 & -16 . & 2451811.73493 & $18.3 \pm 16.2$ \\
\hline 155.6 & -22 & $\mathbf{J} 23$ & $2>$ \\
\hline 2454167.69909 & $-23.1 \pm 6.9$ & 3963.82187 & 5.5 \\
\hline 2454169.67087 & $-25.3 \pm 6.0$ & 2452203.63065 & \pm 18.7 \\
\hline & -4 & & \\
\hline & & & \\
\hline
\end{tabular}

spectral types are rather late (see Willems \& Kolb 2004, for more details). We therefore claim to have identified 9 PCEBs.

In the first paper of this series (Rebassa-Mansergas et al. 2007), multiple SDSS spectroscopy was used to identify 18 PCEB candidates among 101 WDMS binaries from the SDSS. 
Table 4. Radial velocities measured from the Na I $\lambda \lambda 8183.27,8194.81$ doublet in the Clay/LDSS3 spectra of the two PCEB candidates SDSS J1047+0523 and SDSS J1414-0132.

\begin{tabular}{|c|c|c|c|}
\hline HJD & $R V\left[\mathrm{~km} \mathrm{~s}^{-1}\right]$ & HJD & $R V\left[\mathrm{~km} \mathrm{~s}^{-1}\right]$ \\
\hline \multicolumn{2}{|c|}{ SDSS J1047+0523 } & \multicolumn{2}{|c|}{ SDSS J1414-0132 } \\
\hline 2454237.54059 & $214.7 \pm 10.4$ & 2454237.616516 & $190.1 \pm 11.0$ \\
\hline 2454237.64147 & $0.0 \pm 20.8$ & 2454237.625451 & $192.7 \pm 12.1$ \\
\hline 2454238.48000 & $-127.5 \pm 9.5$ & 2454238.587532 & $-78.8 \pm 11.2$ \\
\hline 2454238.51267 & $-107.0 \pm 17.2$ & 2454238.736750 & $-100.0 \pm 10.5$ \\
\hline 2454238.55507 & $-72.5 \pm 17.1$ & 2454239.576845 & $4.8 \pm 14.9$ \\
\hline 2454238.66262 & $240.7 \pm 9.8$ & 2454239.686966 & $160.5 \pm 7.0$ \\
\hline 2454239.54693 & $6.6 \pm 19.8$ & 2454239.752785 & $209.9 \pm 17.2$ \\
\hline 2454239.58773 & $-119.4 \pm 11.9$ & 2454240.498312 & $189.0 \pm 11.8$ \\
\hline 2454239.61468 & $-129.7 \pm 8.8$ & 2454240.569119 & $180.2 \pm 8.8$ \\
\hline 2454239.64270 & $-133.0 \pm 10.0$ & 2454240.579801 & $181.0 \pm 11.0$ \\
\hline 2454240.46501 & $-31.9 \pm 11.6$ & 2454240.671845 & $37.0 \pm 7.7$ \\
\hline 2454240.51077 & $109.5 \pm 10.5$ & 2454240.696022 & $8.1 \pm 10.4$ \\
\hline 2454240.59002 & $218.7 \pm 10.5$ & 2454240.753426 & $-64.1 \pm 11.4$ \\
\hline 2454240.64421 & $162.7 \pm 11.7$ & 2454240.762373 & $-62.6 \pm 12.0$ \\
\hline 2454240.65411 & $163.4 \pm 9.6$ & 2454240.790358 & $-108.4 \pm 7.9$ \\
\hline & & 2454240.801040 & $-119.1 \pm 13.8$ \\
\hline
\end{tabular}

Due to the low spectral resolution of SDSS spectra and the fact that in most of those 101 WDMS binaries, only two SDSS spectra are available, the resulting PCEB fraction of $\sim 15 \%$ certainly has to be considered as a lower limit to the true value. In this paper we identified 9 PCEBs among 26 SDSS WDMS binaries, which gives a PCEB fraction of $35 \pm 12 \%$, which is - as expected - substantially higher than our previous lower limit. The increased PCEB detection rate is probably the result of both the higher resolution of VLT/FORS2 and the fact that 3-4 spectra are available for most systems.

However, in the case that more than 2 spectra are available there also exists a certain probability sampling similar orbital phases, and the observations presented here also have a finite spectral resolution. In the context of our project on PCEB studies, it is important to understand possible selection effects in the established PCEB sample. We therefore developed a MonteCarlo code to estimate the detection probability among SDSS WDMS binaries as a function of orbital period. For the purpose of this specific paper, we limited our analysis to the following assumptions: a mean radial velocity resolution of $6 \mathrm{~km} / \mathrm{s}$, stellar masses of $M_{\mathrm{wd}}=0.6 M_{\odot}$ and $M_{\mathrm{sec}}=0.3 M_{\odot}$, and uniformly distributed inclinations. For an assumed orbital period, we randomly selected the inclination, orbital phase, and times of observations. The only constraint on the last was that the time differences should exceed $12 \mathrm{~h}$ and that all observations are performed in one semester. The randomly selected values we are then used to calculate radial velocity differences. Repeating this exercise $10^{4}$ times and counting the number of cases with a $3 \sigma$ (alternatively 1 or $2 \sigma$ ) radial velocity variation gives the probability of detecting a PCEB with the assumed orbital period. Figure 8 shows the results we obtain assuming that 2 or 3 spectra have been taken in one semester. In both cases the detection probability of different significance levels of radial velocity variations is shown. Of course, our strategy leads to a certain bias towards shorter orbital periods (and higher inclinations) because strong radial velocity variations are more likely to be detected in these systems. However, with three randomly taken VLT/FORS2 spectra, our $3 \sigma$ criterion is still $\sim 15-30 \%$ sensitive to PCEBs with periods in the range of $\sim 20-60$ days. PCEBs with orbital periods longer than 70 days may hide among the systems with

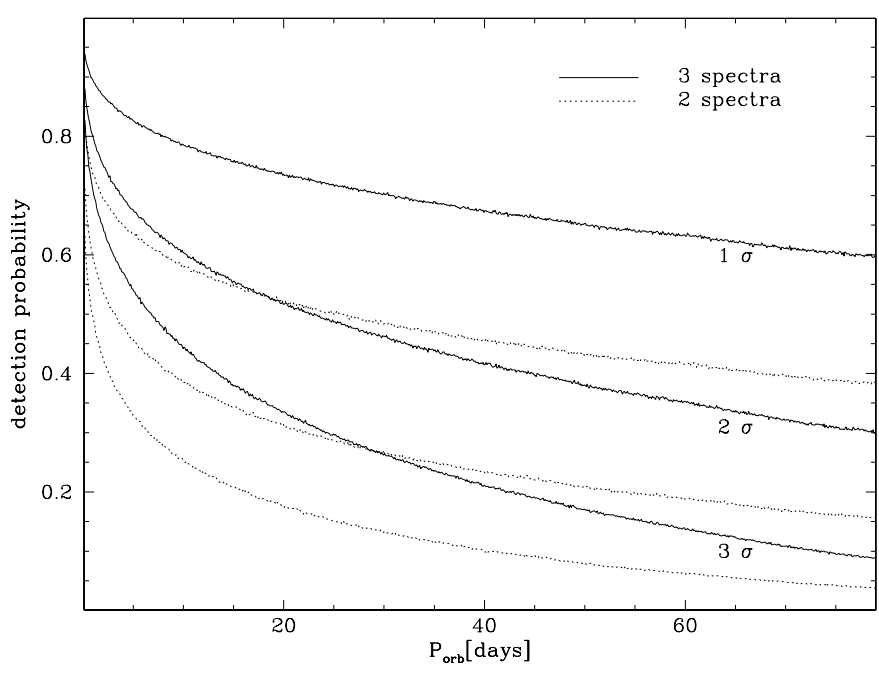

Fig. 8. Detection probability versus orbital period for 2 and 3 spectra taken randomly in one semester but not in the same night. We here assumed $M_{\mathrm{wd}}=0.6 M_{\odot}$ and $M_{\mathrm{sec}}=0.3 M_{\odot}$. The $1-3 \sigma$ labels refer to the detection level of radial velocity variations. Apparently, using our $3 \sigma$ criterion to select strong PCEB candidates for follow-up observations leads to final a sample that is biased toward shorter orbital periods. However, only 3 spectra are needed to identify $15-30 \%$ of the PCEBs with longer orbital periods ( $P_{\text {orb }} \gtrsim 20$ days) and our strategy can therefore certainly be used to also constrain the orbital period distribution of long orbital period PCEBs.

weak $(2 \sigma)$ radial velocity variations, and will require additional spectroscopy to be unambiguously identified. In general, Fig. 8 clearly shows that the fraction of PCEBs among SDSS WDMS binaries of $35 \pm 12 \%$ obtained here still represents a lower limit. However, as mentioned in the introduction, the relative number of PCEBs may be a function of the spectral type of the secondary.

\subsection{Dependence on the secondary stars spectral type}

Recently, Politano \& Weiler (2006) suggested a test for the disrupted magnetic braking scenario, i.e. the hypothesis that magnetic braking is not active in PCEBS that contain fully convective companion stars. Their calculations predict that the relative number of PCEBs among WDMS binaries should drastically increase for secondary star masses below $M_{\mathrm{sec}}=0.35 M_{\odot}$. Our results are consistent with that prediction. Among 9 WDMS binaries with spectral type M3 or earlier we find only one strong PCEB candidate, while the PCEB identification rate among WDMS binaries containing later secondaries (M4-M6) is $8 / 17$. According to the Poisson statistic, the relative numbers of PCEBs among SDSS WDMS binaries are $0.111 \pm 0.111$ (for M0-M3) and $0.471 \pm 0.166$ (for M4-M6). Combining these values, we obtained a significance of $1.8 \sigma$ for the hypothesis of having two different distributions. Interestingly, a potential difference between the secondary star spectral types in close and wide WDMS binaries has also been suggested by Farihi et al. (2005), who found, subject to small number statistics, that the median secondary spectral type of close binaries is one spectral class later than that of the wide binaries. While keeping in mind the well-known problems resulting from low number statistics, these results may represent first indications of a discontinuity in the relative number of PCEBs among WDMS binaries, as predicted by Politano \& Weiler (2006). However, more observations 
are certainly needed to confirm or disprove the observed tendency.

\subsection{Predicted evolution of SDSS J1047+0523 and SDSS J1414-0132}

Knowing the mass and effective temperature of the white dwarf, its orbital period, and at least reasonable estimates for the mass and the radius of the secondary, one can determine the age of the PCEB, the orbital period of the future semi-detached system (i.e. a cataclysmic variable), and the time the PCEB evolution takes for a given system. To that end, we here first follow Schreiber \& Gänsicke (2003) by using the white dwarf cooling tracks of Wood (1995) to determine the white dwarf cooling ages of SDSS J1047+0523 and SDSS J1414-0132. Using the white dwarf parameters given in Table 1 and interpolating the cooling tracks we get $t_{\text {cool }} \sim 2.1 \pm 1 \times 10^{8}$ yrs and $t_{\text {cool }} \sim 3.9 \pm 1.1 \times 10^{8}$ yrs for SDSS J1047+0523 and SDSS J1414-0132, respectively.

The determination of the mass and the radius of the secondary is much more challenging, as the inclination of these two systems is unconstrained. We use the empirical spectral type-radius-mass (Sp-R-M) relation derived in Paper I (Sect. 3.4) to estimate values of the mass and radius of the secondary. Apparently, the scatter around this empirical relation is quite large (Paper I, Fig. 7), so the obtained values should generally be considered as uncertain. However, in the case of SDSS J14140132 and SDSS J1047+0523, the distance estimates based on the white dwarf $\left(d_{\mathrm{WD}}\right)$ and those derived using the empirical Sp-R$M$ relation agree quite well, which suggests that the values are plausible (for a detailed discussion of the spectral type-massradius relation see Paper I). As both secondaries have the spectral type M5, the empirical relation gives $M_{\mathrm{sec}}=0.216 M_{\odot}$ and $R_{\mathrm{sec}}=0.258 R_{\odot}$. For our theoretical analysis, we adopt rather broad ranges of possible values to account for the described uncertainties, i.e. $M_{\mathrm{sec}}=0.20-0.23 M_{\odot}$ and $R_{\mathrm{sec}}=0.2-0.3 R_{\odot}$.

According to the standard scenario of $\mathrm{CV}$ evolution, magnetic braking is disrupted when the secondary stars becomes fully convective (e.g. Verbunt \& Zwaan 1981); hence, gravitational radiation is the only angular momentum loss mechanism acting in systems with a low-mass $\left(M_{\mathrm{sec}} \sim 0.3 M_{\odot}\right)$ secondary star. The evolution of SDSS J1047+0523 and SDSS J1414-0132 according to this scenario and taking the uncertainties discussed above into account is shown in Fig. 9. Both systems will become $\mathrm{CVs}$ in or slightly below the $2-3 \mathrm{~h}$ orbital period gap, and for both systems the evolution will take longer than a Hubble-time. Therefore, according to Schreiber \& Gänsicke (2003), they are different to the progenitors of the current $\mathrm{CV}$ population.

\section{Summary}

We have obtained ESO-VLT/FORS2 spectroscopy for 26 WDMS binaries found in SDSS. Clear radial velocity variations led to identifying of 9 strong PCEB candidates, which gives a hit-rate of $35 \pm 12 \%$. We analysed the PCEB detection probability of our strategy and conclude that (although a bias towards short orbital period systems is inevitable) our survey is sensitive to the predicted long orbital period distribution of PCEBs. While we find 8 PCEB candidates among 17 systems with M4 or later secondary stars, only one PCEB candidate could be identified among 9 WDMS binaries containing earlier secondary stars. Although suffering from low number statistics, one may interpret these results as a first indication of a discontinuity in the dependence of the relative number of PCEBs among WDMS binaries on the spectral type of the secondary.

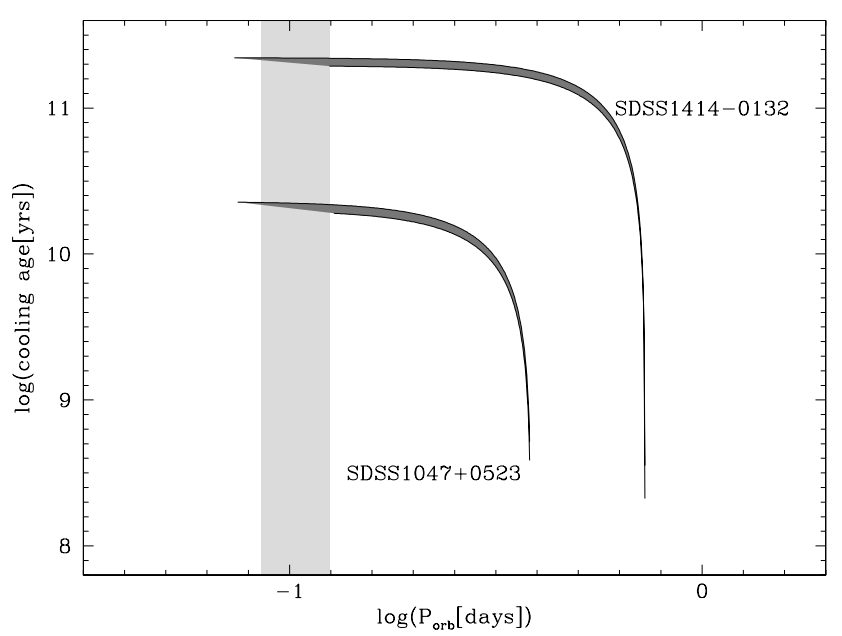

Fig. 9. The PCEB evolution of SDSS J1047+0523 and SDSS J14140132. Starting at their current orbital periods of $P_{\text {orb }}=17.48 \mathrm{~h}$ and $P_{\text {orb }}=9.17 \mathrm{~h}$, the systems evolve towards a shorter orbital period due to angular momentum loss by gravitational radiation. The companion stars in SDSS J1047+0523 and SDSS J1414-0132 fill their Rochelobes in $1.7 \pm 0.3 \times 10^{10} \mathrm{yrs}$ and $1.8 \pm 0.2 \times 10^{11} \mathrm{yrs}$, respectively, and turn these PCEBS into cataclysmic variables. This will happen at very similar orbital periods, $0.090 \pm 0.013$ days for SDSS J1047+0523 and $0.085 \pm 0.015$ for SDSS J1414-0132.

From Magellan-Clay/LDSS3 spectroscopy we determined that the orbital periods for two of the PCEBs identified here, SDSS J1047+0523 and SDSS J1414-0132, are 9.17 h and $17.48 \mathrm{~h}$, respectively. Both systems will enter the semi-detached CV state in or below the orbital period gap. This evolution will last longer than the Hubble time, and both systems are therefore different than the progenitors of the current $\mathrm{CV}$ population.

Acknowledgements. M.R.S. acknowledges support from FONDECYT (grant 1061199), DIPUV (project 35), the Centre of Astrophysics in Valparaiso, and the DLR under grant number No. FKZ 50 OX 0404. J.S. acknowledges financial support from PPARC in the form of a postdoctoral research assistant position.

\section{References}

Adelman-McCarthy, J. K., Agüeros, M. A., Allam, S. S., et al. 2007, ApJS, 172, 634

Adelman-McCarthy, J. K., Agüeros, M. A., Allam, S. S., et al. 2008, ApJS, 175, 297

Andronov, N., Pinsonneault, M., \& Sills, A. 2003, ApJ, 582, 358

Aungwerojwit, A., Gänsicke, B. T., Rodríguez-Gil, P., et al. 2007, A\&A, 469, 297

Beer, M. E., Dray, L. M., King, A. R., \& Wynn, G. A. 2007, 375, 1000 Bergeron, P., Wesemael, F., Lamontagne, R., et al. 1995, ApJ, 449, 258 Eisenstein, D. J., Liebert, J., Harris, H. C., et al. 2006, ApJS, 167, 40 Farihi, J., Becklin, E. E., \& Zuckerman, B. 2005, ApJS, 161, 394 Fontaine, G., Brassard, P., \& Bergeron, P. 2001, PASP, 113, 409 Gänsicke, B. T., Araujo-Betancor, S., Hagen, H.-J., et al. 2004, A\&A, 418, 265 Horne, K. 1986, PASP, 98, 609

Howell, S. B., Nelson, L. A., \& Rappaport, S. 2001, ApJ, 550, 897

King, A. R. 1988, QJRAS, 29, 1

Koester, D., Napiwotzki, R., Voss, B., Homeier, D., \& Reimers, D. 2005, A\&A, 439, 317

López-Morales, M. 2007, ApJ, 660, 732

Marsh, T. R. 1989, PASP, 101, 1032

Marsh, T. R., Robinson, E. L., \& Wood, J. H. 1994, MNRAS, 266, 137

Morales-Rueda, L., Marsh, T. R., Maxted, P. F. L., et al. 2005, 359, 648

Morris, M. 1981, ApJ, 249, 572

Nelemans, G. \& Tout, C. A. 2005, MNRAS, 356, 753

Nelemans, G., Verbunt, F., Yungelson, L. R., \& Portegies Zwart, S. F. 2000, A\&A, 360, 1011

Osterbrock, D. E., Fulbright, J. P., Martel, A. R., et al. 1996, PASP, 108, 277 
Osterbrock, D. E., Fulbright, J. P., \& Bida, T. A. 1997, PASP, 109, 614

Paczyński, B. 1976, in Structure and Evolution of Close Binary Systems, IAU Symp., 73, 75

Pinsonneault, M. H., Andronov, N., \& Sills, A. 2002, in The Physics of Cataclysmic Variables and Related Objects, ed. B. T. Gänsicke, K. Beuermann, \& K. Reinsch, ASP Conf. Ser., 261, 208

Politano, M., \& Weiler, K. P. 2006, ApJ 641, L137

Rebassa-Mansergas, A., Gaensicke, B. T., Rodriguez-Gil, P., Schreiber, M. R., \& Koester, D. 2007, ArXiv e-prints, 707

Ribas, I. 2006, Ap\&SS, 304, 89

Scargle, J. D. 1982, ApJ, 263, 835

Schreiber, M. R., \& Gänsicke, B. T. 2003, A\&A, 406, 304

Schreiber, M. R., Nebot Gomez-Moran, A., \& Schwope, A. D. 2007, in ASP Conf. Ser., 372, ed. A. Napiwotzki, \& M. R. Burleigh, 459

Shimansky, V., Sakhibullin, N. A., Bikmaev, I., et al. 2006, A\&A, 456, 1069

Silvestri, N. M., Lemagie, M. P., Hawley, S. L., et al. 2007, ArXiv e-prints, 704
Southworth, J., Gänsicke, B. T., Marsh, T. R., et al. 2006, MNRAS, 373, 687 Southworth, J., Marsh, T. R., Gänsicke, B. T., et al. 2007, MNRAS, 382, 1145 Taam, R. E., \& Bodenheimer, P. 1989, ApJ, 337, 849

Taam, R. E., \& Ricker, P. M. 2006, ArXiv Astrophysics e-prints

Taam, R. E., \& Sandquist, E. L. 2000, ARA\&A, 38, 113

Tappert, C., Gänsicke, B. T., Schmidtobreick, L., et al. 2007, A\&A, 474, 205

van den Besselaar, E. J. M., Roelofs, G. H. A., Nelemans, G. A., Augusteijn, T., \& Groot, P. J. 2005, 434, L13

van den Besselaar, E. J. M., Greimel, R., Morales-Rueda, L., et al. 2007, 466, 1031

Verbunt, F., \& Zwaan, C. 1981, A\&A, 100, L7

Webbink, R. F. 1984, ApJ, 277, 355

Webbink, R. F. 2007, ArXiv e-prints, 704

Willems, B., \& Kolb, U. 2004, A\&A, 419, 1057

Wood, M. A. 1995, in White Dwarfs, ed. D. Koester, \& K. Werner (Heidelberg: Springer), Lect. Notes Phys., 443, 41 